\title{
Effect of annealing on conductivity in XLPE mid-voltage cable insulation
}

\author{
F. Frutos ${ }^{\mathrm{a}}$, M. Acedo ${ }^{\mathrm{a}}$, M. Mudarra ${ }^{\mathrm{b}}$, J. Belana ${ }^{\mathrm{b}, *}$, J. Òrrit $^{\mathrm{b}}$, J.A. Diego ${ }^{\mathrm{b}}$, \\ J.C. Cañadas ${ }^{\mathrm{b}}$, J. Sellarès ${ }^{\mathrm{b}}$ \\ ${ }^{a}$ Dpto. de Física Aplicada I, ETSII, Universidad de Sevilla, Avda. Reina Mercedes s/n, 41012 Sevilla, Spain \\ ${ }^{\mathrm{b}}$ Dept. de Física i Enginyeria Nuclear, ETSEIAT, Universitat Politècnica de Catalunya, c. Colom 11, 08222 Terrassa, Spain
}

\section{Introduction}

Polyethylene (PE) is a non-polar polymer. It is found in semi-crystalline form at room temperature. The crystalline fraction is formed when the material is cooled down from the melt. The lamellae structure formed during solidification depends on the characteristics of the thermal treatment and the conditions of crystallization. In the usual conformation, lamellae grow radially, resulting in spherulites, which are composed of both crystalline and amorphous regions.

Due to the non-polar character of PE, conduction processes should be associated with the presence of free

*Corresponding author.

E-mail address: juan.belana@upc.edu (J. Belana). charge carriers. Nevertheless, polar contribution may arise from the existence of carbonyl groups $\mathrm{C}=\mathrm{O}$ that are produced by oxidation of $\mathrm{PE}$, although there may also exist polar impurities.

In industrial applications, PE is commonly used as an electrical insulator. Specifically, it constitutes the base compound for cable insulation manufacturing due to its outstanding insulating characteristics and its low price. In the standard configuration of mid-voltage (MV) cables (up to $25 \mathrm{kV}$ service voltage), the PE insulation is covered by two coaxial cylindrical semiconducting (SC) screens which are both extruded simultaneously during fabrication, resulting in a concentric three layer system: SC-insulation-SC. The composition of these screens (tipically a blend of PE, ethylene vinyl acetate and carbon black) results in a low-resistivity material that prevents discharges 
on the surface of the insulation that may damage it. In addition, the PE insulation is subjected to a crosslinking process so that it finally results in crosslinked PE (XLPE). This process improves its properties; particularly it enhances its chemical and mechanical resistance, allowing a higher service temperature.

It is well known that PE conductive properties are conditioned by its morphology [1-8] and that insulation degradation can be related to volume space charges [9]. Particularly, conduction and space charge formation in low-density PE were studied including the interval of temperatures of interest for cable diagnostics [10]. In the last few years, there has also been a growing interest in the study of insulation properties under the application of DC voltage, the corresponding formation of space charge distributions and its relation to the remaining lifetime of the cable [11-13]. Although it is generally considered that carriers responsible for PE conduction are basically electrons $[13,14]$, ions coming from additives and crosslinking by-products should be considered also.

Current versus voltage $(I-V)$ characteristics studied in LDPE by Stetter [15] at 42,82 and $110^{\circ} \mathrm{C}$, indicate that for low electric fields $(E)$ an ohmic behaviour is observed; for higher electric fields, conduction can be interpreted on the basis of a quadratic law in $E$ which depends on temperature and reveals the existence of traps. Finally, for higher electric fields, a third region can be found where current follows Child's law with full traps, and conduction can be interpreted from general criteria of the theory of space-charge-limited currents (SCLC) [16]. More recently, several authors have also observed that current-voltage characteristics in PE can be explained by SCLC model $[17,18]$. Pélissou et al. [17] studied the isochronal curren$\mathrm{t}$-voltage characteristics in LDPE for a wide temperature range below and above its melting point. They concluded that at low average electrical fields the behaviour is ohmic, but between 5 and $50 \mathrm{MV} / \mathrm{m}$ it becomes highly non-linear, following $J \propto V^{n}$ with $n>2$. This result suggests that an exponential distribution of traps may be present [18]. In another study of $I-V$ characteristics, Mizutani shows that current is limited by the electrode, following a Richardson-Schottky law for high electric fields $\left(E>4 \times 10^{5} \mathrm{~V} / \mathrm{cm}\right)[19]$.

Nath et al. [20] apply the band theory to LDPE by developing a mathematical model based on the hopping of carriers which are injected into amorphous regions. They consider a SCLC and a process of charge-trapping at amorphous-crystalline boundaries. In addition, they suppose that the density of trapping centres is high enough so that the interaction between them results in an effective lowering of the trap depth (Poole-Frenkel effect). In this latter model, the "distance between traps" parameter is introduced and it has a constant value. Even more recently, following the same research theme, it is concluded that electronic transport is bound to thermally activated hopping, which is assisted by electric field with a very low activation energy [21]. From charge distribution studies and, specifically, by applying the electroacoustic pulse technique (PEA), the presence of periodical chargepackets between electrodes can be detected for high electric fields [22].

Referring to the nature and depth of traps, it has been proposed that impurities and/or chain defects could be responsible for their existence in the material. Ieda [23] has performed a detailed study on PE inquiring into both theories.

Thermally stimulated depolarization current technique (TSDC) has been applied to XLPE cable electrets [24,25]. The spectrum obtained (intensity of the depolarization current versus temperature) is complex and it is formed by several peaks associated with the relaxation processes of the conduction mechanisms that are present in the material. A broad current peak can be observed at $105^{\circ} \mathrm{C}$, which has been associated with charge in the crystalline volume [24]. The combination of TSDC and infrared spectroscopy (IR) studies [25] proved that annealing procedures in $\mathrm{MV}$ cables lead to the diffusion of components from the SC screens into the XLPE insulation bulk. Moreover, the defects associated with diffused particles act as trapping centres of injected charge from electrodes, by ionization. This process is clearly prominent at temperatures above the critical temperature, $T_{\mathrm{c}}$, approximately equal to $80^{\circ} \mathrm{C}$, because TSDC current reverses its polarity (from heteropolar to homopolar). Further annealing results in new current polarity inversion, and the current regains its heteropolar character [24]. These changes are in agreement with the fact that diffused components continue to penetrate the insulation volume. In addition, the progressive increase of the heteropolar TSDC curve area indicates a thermally activated generation of ions from an intrinsic source (ions resulting from dissociation of by-products and/or other species present in the insulation).

The aim of this paper is to show the differences in behaviour in conductive processes that take place in XLPE insulation for two different cases: (i) when the insulation retains its original SC screens and, (ii) when the original screens are replaced by metallic electrodes. In this way, we evaluate, by using complementary time/frequency domain techniques, the incidence of component diffusion from SC screens towards the volume of insulation. The temperatures used in this study are close to those for a typical power distribution cable in service conditions. The results obtained may be useful for developing a cable lifetime model on the basis of thermoelectric breakdown.

\section{Experimental}

\subsection{Absorption/resorption currents}

Cable was supplied by General Cable SA (service voltage up to $25 \mathrm{kV}, 4.5 \mathrm{~mm}$ insulation thickness, $1 \mathrm{~mm} \mathrm{SC}$ screens thickness). Samples were cable sections of $7 \mathrm{~cm}$ length. In the case of XLPE cylinders, inner and outer SC screens 
were removed by using a mechanical lathe and they were replaced by adapted copper electrodes for an optimal fitting to the inner and outer surfaces of the XLPE insulation. This procedure allowed us to carry out measurements of the electrical properties of the XLPE insulation, avoiding the effects of the SC screens. In the case of cable samples only the outer SC screen was partially removed, so that a centred ring of $2 \mathrm{~cm}$ width was left. This ring and the inner screen were used as electrodes. It is well known that reliable measuring of high impedance insulation requires very careful guarding and shielding of the measured object. This is of particular importance when measuring small samples [26] or short cables [27]. One metallic ring on each side of the outer electrode, $2 \mathrm{~mm}$ aside of the latter, were used as guard electrode in order to avoid the effect of surface conductivity and to avoid the dispersion of the field lines. The comparison of the results obtained from both kinds of sample allowed us to study the effect of the SC screens during the treatments.

Conductivity $(\sigma)$ was determined by means of equation $[28,29]$

$\sigma(t) \approx \frac{\varepsilon_{0}}{C_{0} U}\left(I_{\mathrm{a}}(t)+I_{\mathrm{r}}(t)\right)$,

where $C_{0}=4.4 \mathrm{pF}$ is the geometrical capacitance of the sample (determined by a Hewlett-Packard impedance analyser model HP-4192a LF), $I_{\mathrm{a}}(t)$ and $I_{\mathrm{r}}(t)$ are the absorption and resorption currents (ARC), respectively, $U$ is the voltage applied to each cable sample and $\varepsilon_{0}$ is the vacuum permittivity. The ARC method provides a convenient way to obtain $\sigma$ because measurements require less time to be performed than with the usual current-voltage characteristics method since there is no need to reach a stationary current.

Previously to any measurement, the surface of the sample was cleaned with ethanol in order to avoid the effect of remains that may come from the mechanization process. The MV cable samples and XLPE cylinders to be measured were placed inside a Carbolyte type PF60 oven (Faraday cage). The oven was furnished with suitable connections for electrical measurements, using low-noise coaxial connectors and cables, suitable for high voltage. Cable and connector junctions were silver soldered. XLPE cylinders and cable samples were placed in a measuring cell inside the oven described above. A K-type thermocouple (Keithley 6517-TP) placed inside the insulation of another identical sample, which was located close to the sample under test, was used to measure the sample temperature. The DC voltage source of a Keithley $6517 \mathrm{~A}$ electrometer was used to polarize the samples. To measure the absorption current a voltage of $1 \mathrm{kV}$ (corresponding to a mean field of $0.22 \mathrm{MV} / \mathrm{m}$ ) was applied to the inner electrode of the samples and the outer electrode was grounded through the aforementioned electrometer. After a period of time, the inner electrode was switched to ground and the resorption current was recorded. The charging period (absorption current) was about the double of the discharging period (resorption current), 2000 and $1000 \mathrm{~s}$ respectively, so that Eq. (1) can be used for conductivity calculations as a good approximation [30]. The background noise was lower than $20 \mathrm{fA}$ in all measurements. The entire setup of measuring instrument and screened oven was also placed inside another Faraday cage in order to prevent measurement fluctuations due to external perturbations. A sketch of the setup can be seen in Fig. 1.

A new sample was used in each set of ARC measurements in order to avoid the effects of the previous measurements. Each sample was subjected to annealing at a temperature $T_{\mathrm{a}}\left(50^{\circ} \mathrm{C}<T_{\mathrm{a}}<100^{\circ} \mathrm{C}\right)$ for a time $t_{\mathrm{a}}$. Several annealing times were used for each temperature. The longest annealing times used at each temperature were determined by the kinetics of the changes observed in the conductivity properties and their order of magnitude was
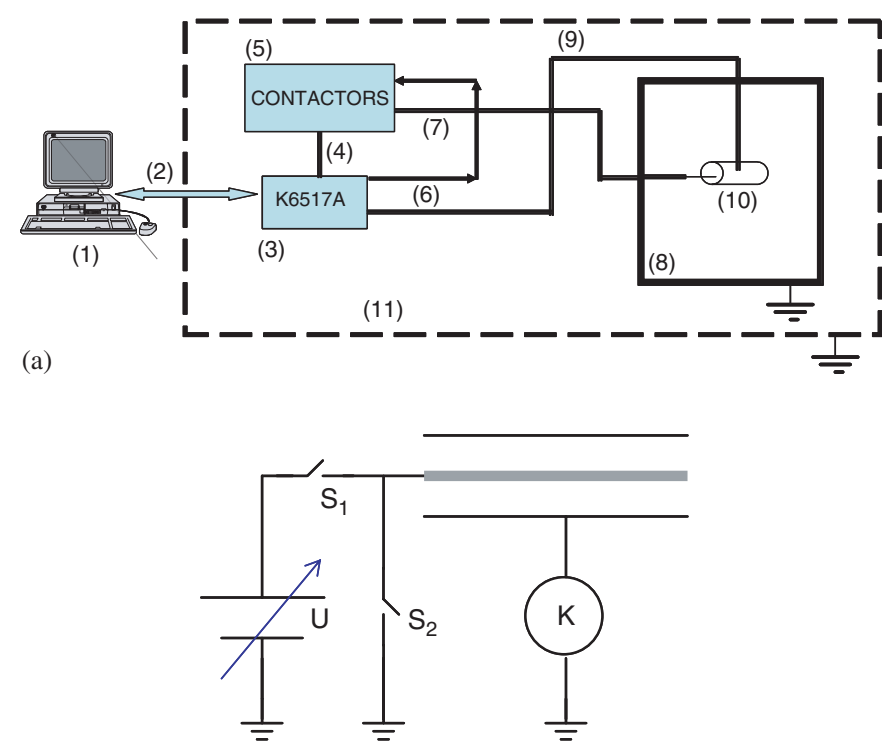

(b)

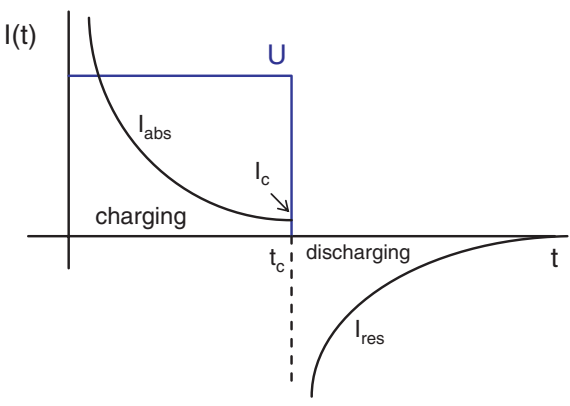

Fig. 1. (a) Experimental setup for absorption/resorption current measurement (block diagram): (1) personal computer (Pentium IV processor); (2) GPIB cables; (3) Keithley 6517A electrometer; (4) DC source output of Keithley 6517A; (5) box of contactors; (6) control of contactors by digital output of Keithley 6517A; (7) high voltage output from contactors; (8) carbolite PF60 oven; (9) coaxial measuring cable; (10) sample; (11) Faraday cage $(2 \mathrm{~m} \times 1 \mathrm{~m} \times 1 \mathrm{~m})$. (b) Simplified sketch of experimental setup for absorption/resorption current measurement and example of measurement: charging period, $0<t<t_{\mathrm{c}}(\mathrm{S} 1 \mathrm{ON}, \mathrm{S} 2 \mathrm{OFF})$ and discharging period, $t>t_{\mathrm{c}}(\mathrm{S} 1 \mathrm{OFF}, \mathrm{S} 2 \mathrm{ON}) ; I_{\mathrm{abs}}$, absorption current; $I_{\text {res }}$, resorption current; $I_{\mathrm{c}}$, conduction current; $t_{\mathrm{c}}$, charging time; $K$, electrometer; $U$, charging voltage. 
tipically $10^{2}-10^{3} \mathrm{~h}$. Using the procedure described in Fig. 1(b) to obtain the ARC values and Eq. (1) to calculate the conductivity, conductivity measurements were performed for several annealing times, in order to determine the evolution of cable conduction up to an eventual stabilization. Nevertheless, a total stabilization was never observed, although, in its place, we could confirm the existence of oscillations around a quasi-stationary value.

\subsection{Dynamic electrical analysis}

Frequency domain characterization was performed via dynamic electric analysis (DEA), using a dielectric spectrometer BDS40 with a Novotherm temperature control system manufactured by Novocontrol, in conditions of isothermal annealing. The samples were $150 \mu \mathrm{m}$-thick XLPE films of $2 \mathrm{~cm}$ diameter, which were cut from a MVC using a lathe with a tool designed for this purpose, so that a $2 \mathrm{~cm}$ width ribbon could be obtained. We performed frequency sweeps between $0.01 \mathrm{~Hz}$ and $1 \mathrm{MHz}$ for different annealing periods of time ranging from 0 to $72 \mathrm{~h}$. Real and imaginary parts of the conductivity were recorded as a function of the frequency for each annealing time and annealing temperature value in order to carry out the discussion of the results.

\section{Results and discussion}

\subsection{Time domain measurements (absorption/resorption currents)}

Experimental results have been grouped by temperature of measurement and annealing, i.e., whether these were carried out at temperatures $T_{\mathrm{a}}$ below or above the critical temperature, $T_{\mathrm{c}}=80^{\circ} \mathrm{C}$. We have arranged experimental data this way in order to emphasize the noticeable observed differences in the evolution of conductivity versus annealing time [31].

\subsubsection{Annealing below $T_{\mathrm{c}}$}

For both cable samples and XLPE cylinders (samples without SC screens), the general behaviour of conductivity versus annealing time consisted in a continuous decrease. This decreasing trend was associated with different levels of conductivity oscillations until reaching an apparent quasi-stationary state after a period of several days. Fluctuations in conductivity continued at least for our total period of annealing time $\left(t_{\mathrm{a}}>1\right.$ month, in the case of $T_{\mathrm{a}}=50^{\circ} \mathrm{C}$, for a XLPE cylinder, as it is shown in Fig. 2), leading to a quasi-stationary state with fluctuations at about the 4th day of measurements. These fluctuations are more noticeable in the case of XLPE cylinder with copper electrodes, especially at low temperatures (Fig. 2). They could be attributed to problems at the electrical contacts. Even considering the slight variations in the nature and geometry of our samples, it is nearly impossible to achieve a perfect fitting between copper electrodes and

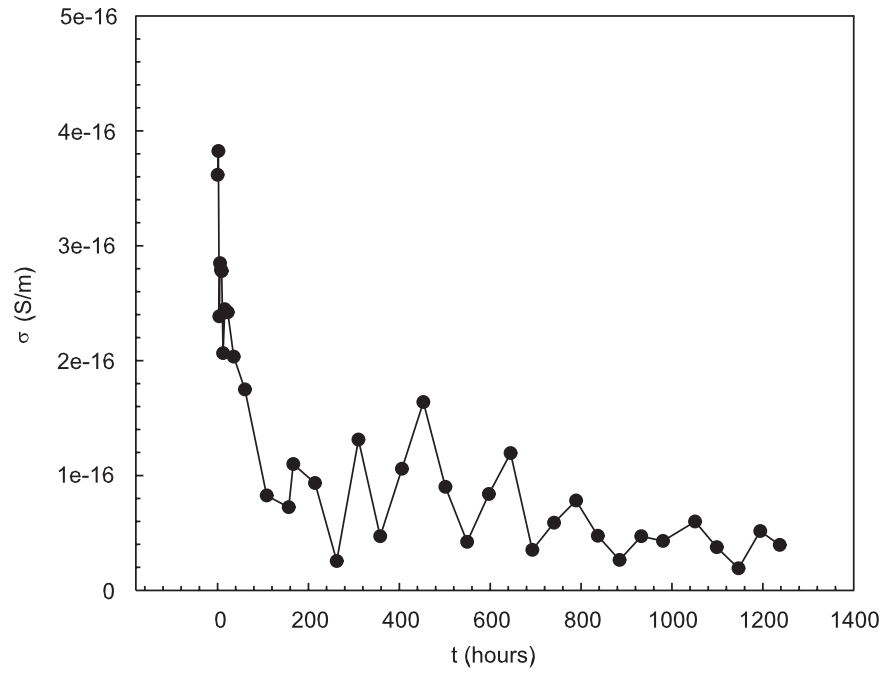

Fig. 2. Evolution of conductivity with annealing time for a XLPE cylinder (cable sample without semiconducting screens) at $50^{\circ} \mathrm{C}$ in isothermal conditions, corresponding to cable $\mathrm{C} 5$.

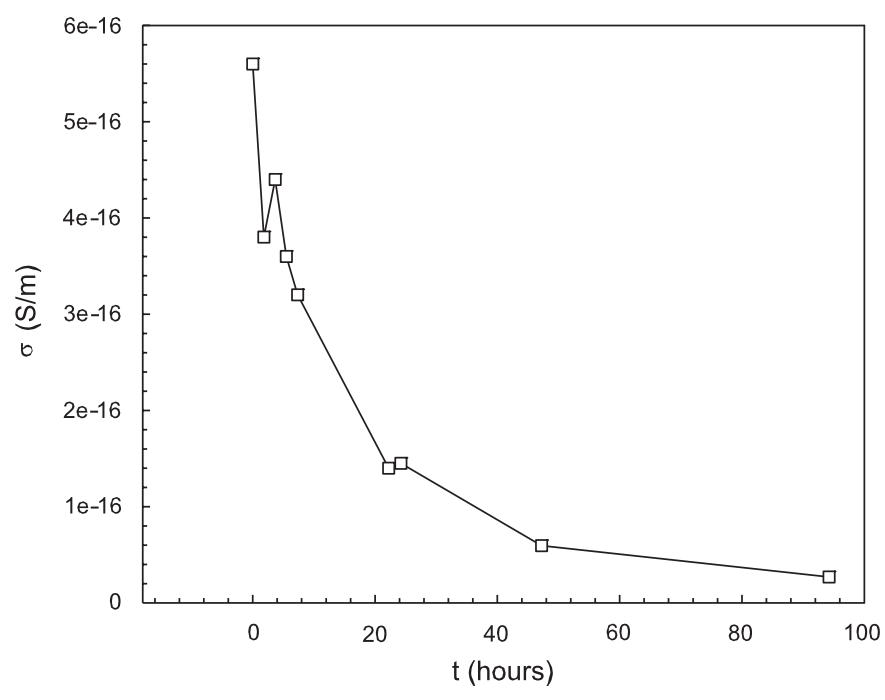

Fig. 3. Evolution of conductivity with annealing time for a cable sample ("complete" cable sample with semiconducting screens) at $50{ }^{\circ} \mathrm{C}$ in isothermal conditions, corresponding to cable C5.

XLPE. This fact probably leads to charge injection (corona effects, partial discharges, etc.) that occasionally may cause sharp fluctuations in the instantaneous values of our measurements.

In Fig. 3, also for $T_{\mathrm{a}}=50^{\circ} \mathrm{C}$, conductivity measurements for a cable sample (cable sample with SC screens) are shown. Due to the clear homogeneity in the decreasing conductivity trend, the measurement period was restricted to less than 4 days. Only during the very first hours of measurement could conductivity fluctuations be detected.

The variations of conductivity in both types of samples (cable samples and XLPE cylinders) for annealing temperatures of 60 and $70^{\circ} \mathrm{C}$ are depicted in Figs. 4 and 5 . Their behaviour is not very different from the conductivity evolutions described for $50^{\circ} \mathrm{C}$. In conclusion, for $T_{\mathrm{a}}<T_{\mathrm{c}}$, 


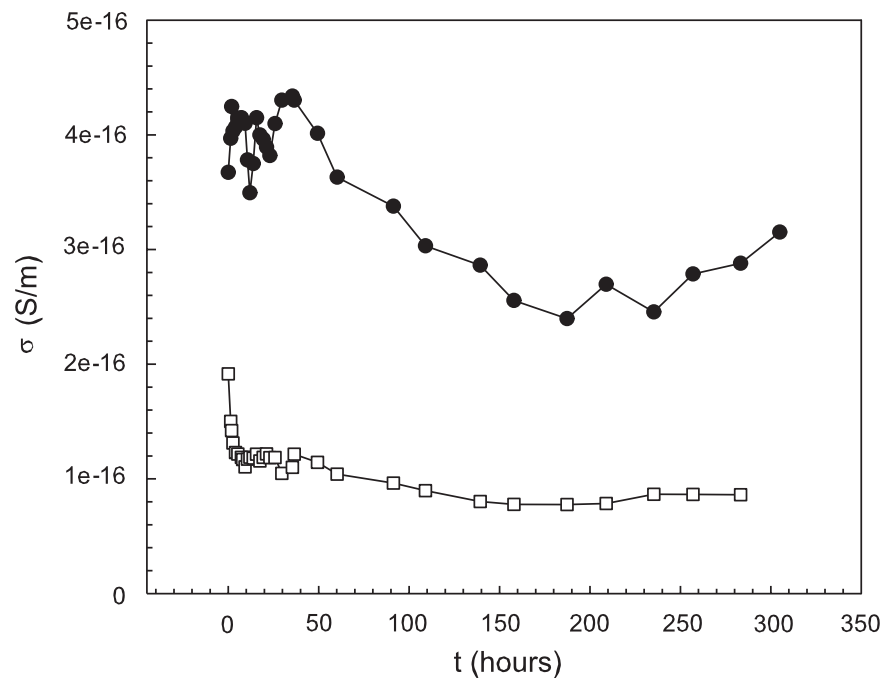

Fig. 4. Evolution of conductivity with annealing time for a cable sample $(\square)$ and a XLPE cylinder $(\bullet)$ at $60^{\circ} \mathrm{C}$ in isothermal conditions.

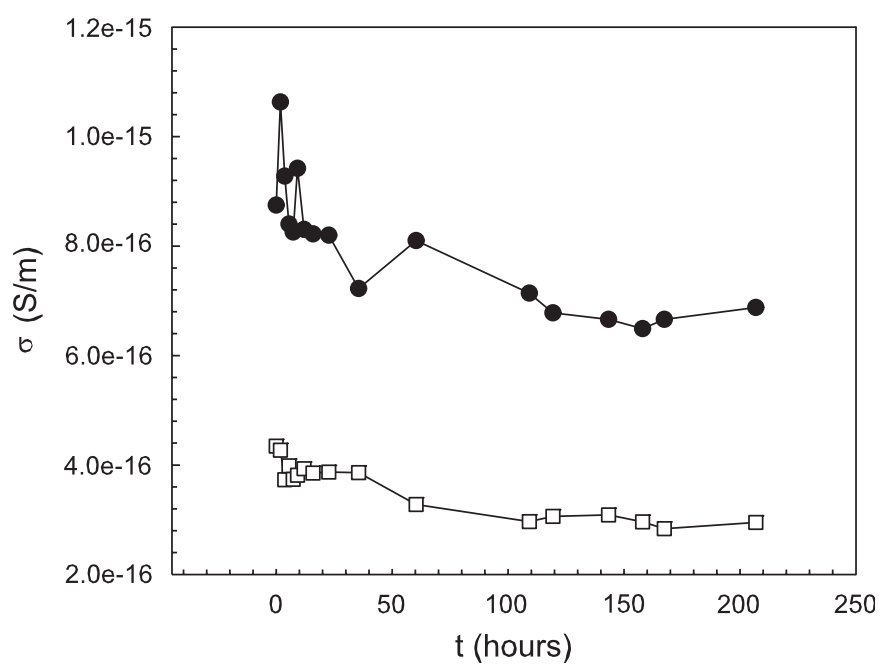

Fig. 5. Evolution of conductivity with annealing time for a cable sample $(\square)$ and a XLPE cylinder $(\bullet)$ at $70{ }^{\circ} \mathrm{C}$ in isothermal conditions.

we determine that the measured conductivity when using SC screens (cable samples) is clearly smaller than conductivity obtained when using copper electrodes (XLPE cylinders). It can be noted that the higher the annealing temperature, the lower the relative variation of the conductivity of the material during the annealing process.

\subsubsection{Annealing above $T_{\mathrm{c}}$}

In Figs. 6-8, we depict the variations in conductivity $(\sigma)$ as a function of the annealing time for temperatures ranging from 80 to $100^{\circ} \mathrm{C}$. Initially, for both sample types, $\sigma=f\left(t_{\mathrm{a}}\right)$ decreases quickly until a minimum value is measured, and thereafter, $\sigma$ begins to increase. From then on, conductivity evolves very differently for the cable sample with SC electrodes and for the XLPE cylinder with copper electrodes.

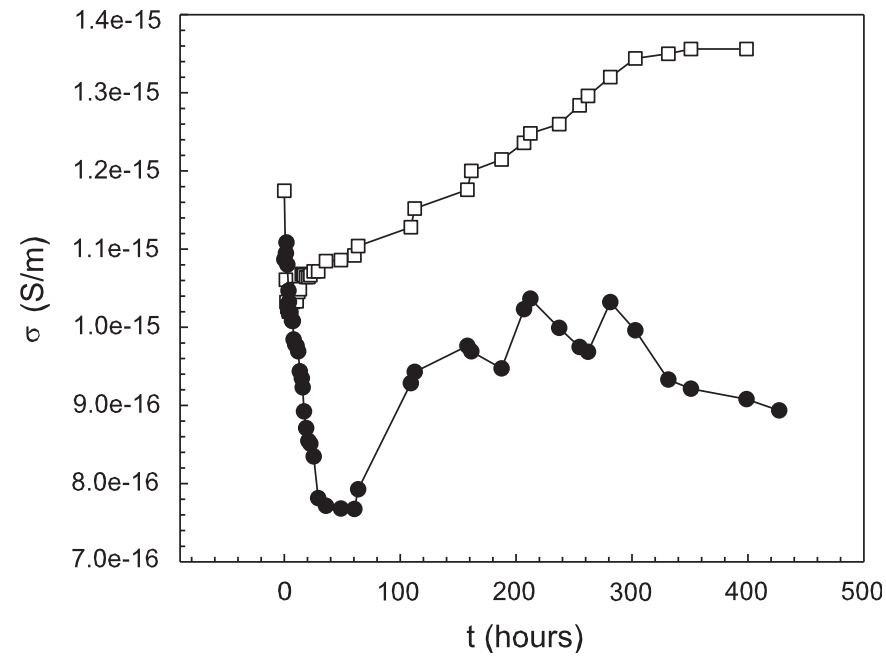

Fig. 6. Evolution of conductivity with annealing time for a cable sample $(\square)$ and a XLPE cylinder $(\bullet)$ at $80^{\circ} \mathrm{C}$ in isothermal conditions.

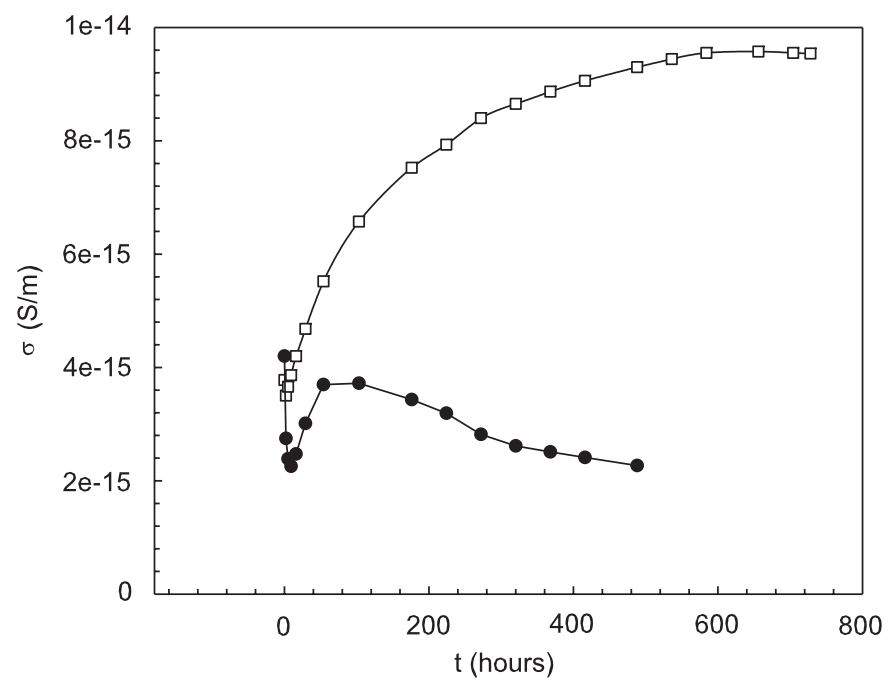

Fig. 7. Evolution of conductivity with annealing time for a cable sample $(\square)$ and a XLPE cylinder $(\bullet)$ at $90^{\circ} \mathrm{C}$ in isothermal conditions.

In the case of copper electrodes (XLPE cylinders), conductivity increases up to a maximum value, and then a monotonically decreasing trend is observed that is accompanied by different levels of fluctuations until a quasi-stationary value is attained. The higher the annealing temperature, the shorter the time required for reaching this maximum value of conductivity. As an example, at $80^{\circ} \mathrm{C}$, it can be observed that this time is several days and fluctuations are very significant, whereas at $100^{\circ} \mathrm{C}$, this time is only of a few days and oscillations are almost imperceptible.

In the case of SC electrodes (cable samples), a monotonic increase in conductivity reaches a quasi-stationary maximum final value. Higher the annealing temperature, the higher is the relative increment of conductivity in comparison with its initial value. This percentage increment is approximately $450 \%$ for $T_{\mathrm{a}}=100{ }^{\circ} \mathrm{C}$ (Fig. 8). 


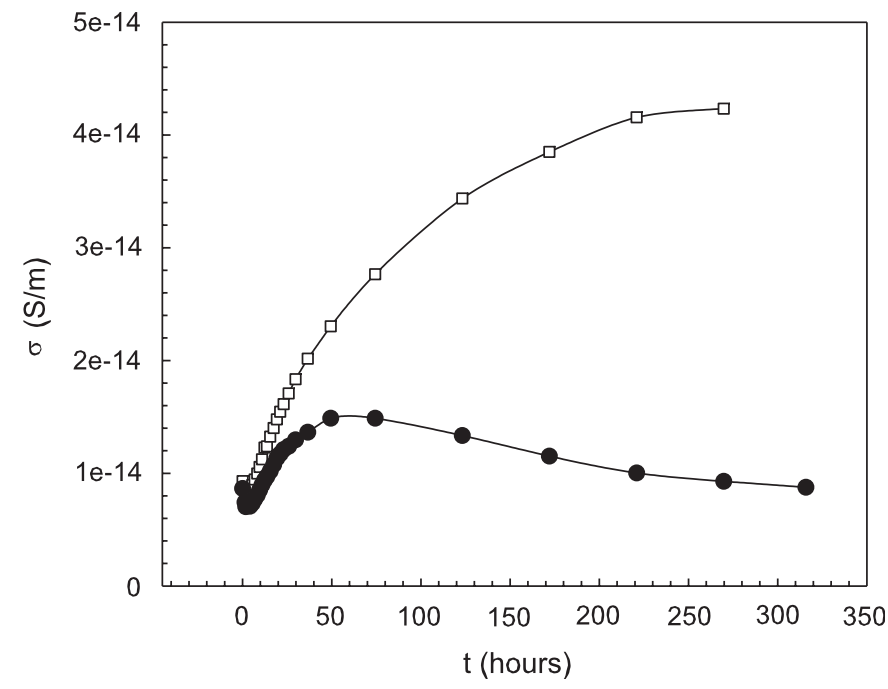

Fig. 8. Evolution of conductivity with annealing time for a cable sample $(\square)$ and a XLPE cylinder $(\bullet)$ at $100^{\circ} \mathrm{C}$ in isothermal conditions.

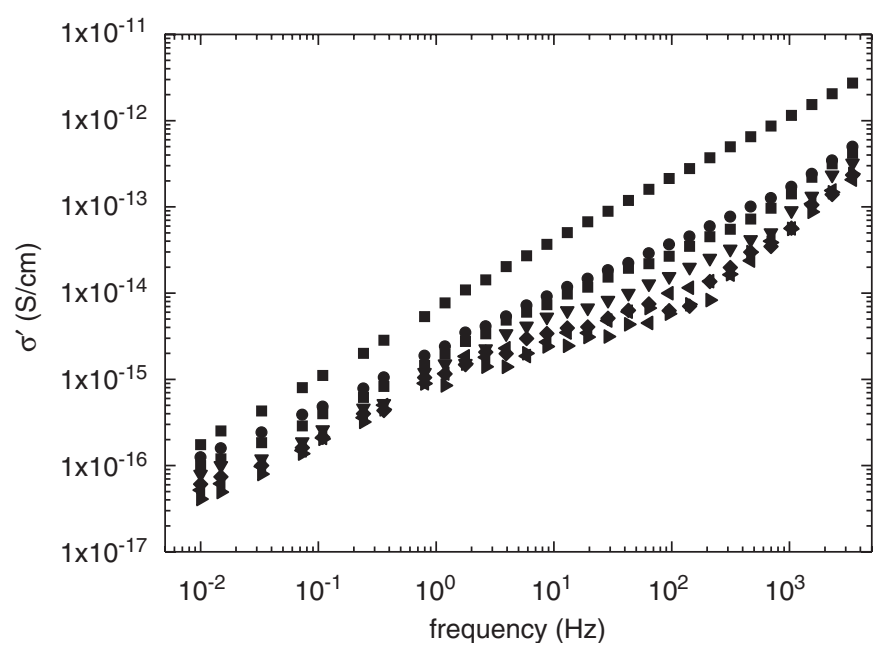

Fig. 9. Variations of the real part of complex conductivity versus frequency by using isochrone parametric curves obtained by DEA technique, for an annealing temperature of $T_{\mathrm{a}}=80^{\circ} \mathrm{C}$ : (口) $t_{\text {annealing }}=0 \mathrm{~h} ; \quad$ (৫) $\quad t_{\text {annealing }}=1 \mathrm{~h} ; \quad(\mathbf{\Delta}) \quad t_{\text {annealing }}=3 \mathrm{~h} ; \quad$ ( $)$ $t_{\text {annealing }}=12 \mathrm{~h} ; \quad(\diamond) \quad t_{\text {annealing }}=24 \mathrm{~h} ; \quad$ (《) $t_{\text {annealing }}=36 \mathrm{~h} ; \quad$ ( $t_{\text {annealing }}=72 \mathrm{~h}$.

\subsection{Frequency domain measurements (DEA)}

Two separate behaviours could be clearly distinguished for the frequency and annealing temperature ranges we used during the conductivity measurements. Some representative results are shown in Figs. 9-11.

For frequencies higher than $1 \mathrm{~Hz}$, we observed a reduction in the real part of complex conductivity versus annealing time for all annealing temperatures ranging from 80 to $105^{\circ} \mathrm{C}$.

For frequencies lower than $1 \mathrm{~Hz}$, two different behaviours were observed. First, for temperatures below $95^{\circ} \mathrm{C}$, the real part of the complex conductivity decreases monotonically with annealing time, and this evolution becomes faster as temperature grows. Furthermore, the range of variation of the conductivity values is also smaller. Secondly, for temperatures above $95^{\circ} \mathrm{C}$, the real part of the complex conductivity initially increases (for example, at $T_{\mathrm{a}}=100^{\circ} \mathrm{C}$, it increases for approximately $12 \mathrm{~h}$ ), but thereafter decreases to previous values. Rising and decreasing periods become shorter as temperature grows.

\subsection{Discussion}

In order to properly interpret the results, it is very important to take into account that DEA measurements are performed only in XLPE samples, but not directly in sections of cable. These samples were cut from a ribbon that had been previously obtained from the insulation of a MV cable, as described in Section 2.1. Therefore, these XLPE DEA samples do not have SC screens as electrodes on their surfaces. For this reason, their results should only be compared with those corresponding to XLPE cylinders (cable samples without SC screens) measured by using the ARC technique.

Secondly, we can confirm that effectively, ARC and DEA results on XLPE samples are consistent based on the conductivity trends with increasing annealing times, both for low and high temperatures. In the case of low temperatures, conductivity always decreases with annealing time and particularly, referring to DEA measurements, this reduction is observed for the real part of complex conductivity along the entire frequency range that was examined (Fig. 9). For higher annealing temperatures, we have also found that experimental results obtained by using both techniques are comparable. At low frequencies (Figs. 10 and 11), the real part of conductivity initially increases with annealing time but afterwards, it decreases again. Particularly, for $T_{\mathrm{a}}=100^{\circ} \mathrm{C}$, an absorption peak at $0.03 \mathrm{~Hz}$ could be present (Fig. 11). Nevertheless, for higher frequencies, there is a continuous reduction of the real part of complex conductivity, which produces a crossover frequency for parametric isochrone curves (curves obtained for different annealing times). This crossover frequency increases with $T_{\mathrm{a}}$.

A global explanation of the different behaviours and variations for conductivity that have been described in previous sections can be established on the basis of the existence of two prevailing types of conduction mechanisms in the material. Both mechanisms are associated with electronic carriers. On the one hand, there is a band conduction mechanism, slightly dependent on temperature (in our range of temperatures), but which is efficient over the entire studied domain of frequencies and annealing measuring times. On the other hand, there is a "hopping" conduction mechanism [32] between localized states, in the mobility gap, which is both temperature and electric field dependent and which is controlled by a Poole-Frenkel mechanism, as we will see shortly. This hopping process is only efficient from a critical temperature value, due to the 


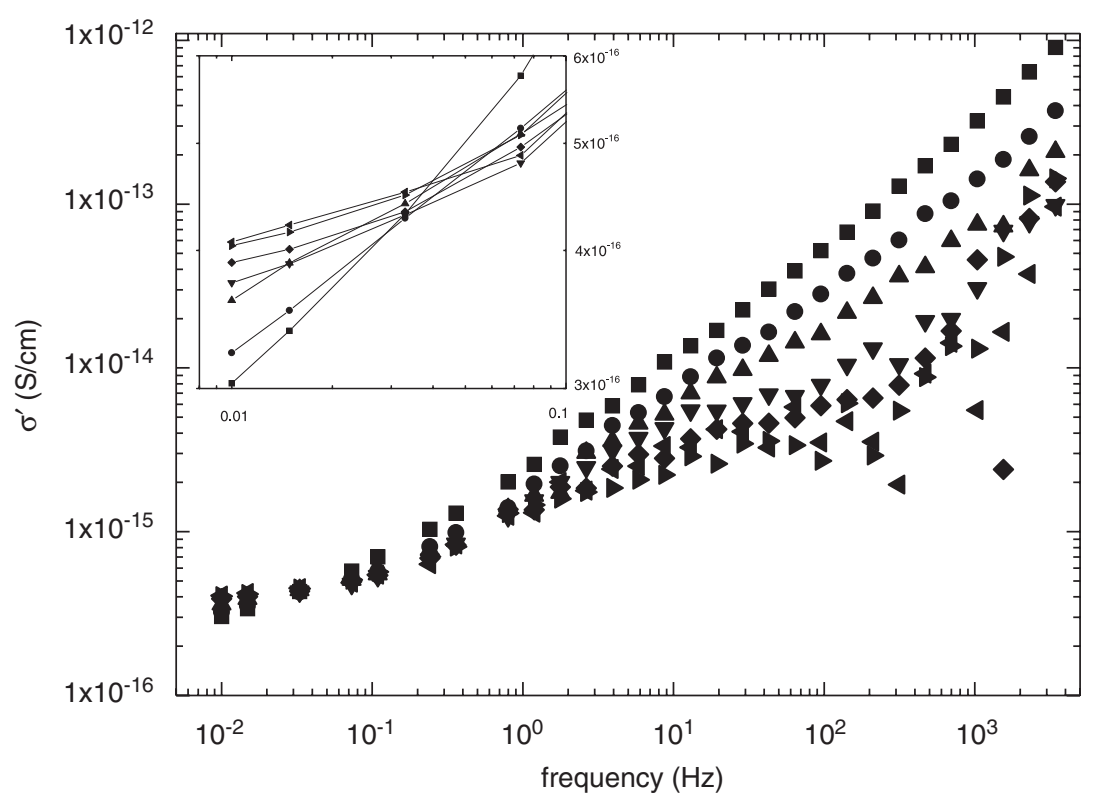

Fig. 10. Variations of the real part of complex conductivity versus frequency by using isochrone parametric curves obtained by DEA technique, for an annealing temperature of $T_{\mathrm{a}}=95^{\circ} \mathrm{C}$ : (田) $t_{\text {annealing }}=0 \mathrm{~h} ;(\bullet) t_{\text {annealing }}=1 \mathrm{~h} ;(\boldsymbol{\Delta}) t_{\text {annealing }}=3 \mathrm{~h} ;(\boldsymbol{\nabla}) t_{\text {annealing }}=12 \mathrm{~h} ;(\bullet) t_{\text {annealing }}=24 \mathrm{~h}$; $(\triangleleft)$ $t_{\text {annealing }}=36 \mathrm{~h} ;(\mathbf{)}) t_{\text {annealing }}=72 \mathrm{~h}$.

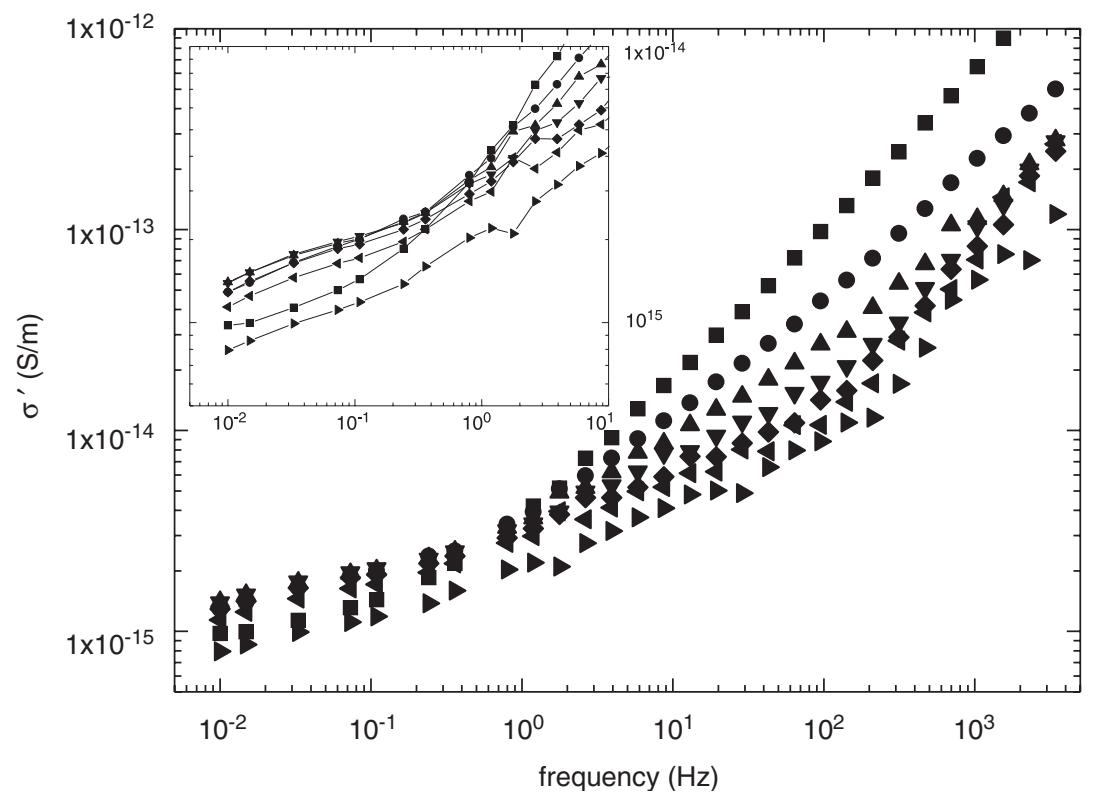

Fig. 11. Variations of the real part of complex conductivity versus frequency by using isochrone parametric curves obtained by DEA technique, for an annealing temperature of $T_{\mathrm{a}}=100^{\circ} \mathrm{C}$ : (⿴) $t_{\text {annealing }}=0 \mathrm{~h} ;(\bullet) t_{\text {annealing }}=1 \mathrm{~h} ;(\boldsymbol{\Delta}) t_{\text {annealing }}=3 \mathrm{~h} ;(\boldsymbol{\nabla}) t_{\text {annealing }}=12 \mathrm{~h}$; ( $) t_{\text {annealing }}=24 \mathrm{~h} ;(\varangle)$

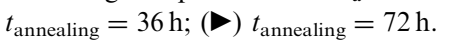

increment of defect concentration associated with diffused components from the SC screens [25]. Dissociation of typical species present in a MV cable insulation, such as crosslinking by-products, may also play a role in this process.

Hopping conduction is greatly enhanced by the diffusion of different components from SC screens to XLPE insulation which implies that there are two determining conditions for hopping conduction to be a prevailing mechanism: (a) the existence of SC screens and (b) temperatures above $T_{\mathrm{c}}$ (i.e. service temperatures of power distribution MV cables) to increase the diffusion of the referenced components $[24,33]$.

\subsubsection{Temperatures below $T_{\mathrm{c}}$}

The assumptions made above can explain the differences in the behaviour of conductivity between XLPE cylinders and cable samples as detected by ARC measurements. 
Below $T_{\mathrm{c}}$, electronic band conduction is the prevailing mechanism, as diffusion at these temperatures can be neglected during annealing procedures [23]. To explain the differences of conductivity observed between the two kinds of samples, we assume that both SC electrodes (cable sample) and copper electrodes (XLPE cylinders) have a blocking character and they limit electric current. This effect is even stronger in the case of SC electrodes (Figs. 4 and 5). For both types of electrodes, their blocking behaviour could be associated with the presence of components diffused from SC screens during the manufacturing process. These components act as trapping centres, and trapped charge limits the flux of electric current. In the case of copper electrodes (XLPE cylinders), mechanical procedures developed to eliminate outer and inner SC screens also remove the most external layers of the insulating XLPE material-where trap concentrations are highest - consequently leading to an important decrease in effective trapping and then resulting in higher values of conduction current and conductivity.

The general trend to reduction of conductivity versus annealing time - especially for short annealing times - can be explained on the basis of a recrystallization process that takes place for this range of temperatures [34]: XLPE crystals suffer a reconfiguration, approaching perfect crystals and possibly of larger dimensions that results in a reduction of conductivity.

These hypotheses also explain the behaviour observed by DEA. We must remember that, by using this technique, we dispose of only XLPE samples (without SC layers), and consequently DEA results should be compared only with ARC measurements performed on XLPE cylinders (with copper electrodes and without SC screens). As we have already shown (Figs. 2, 4 and 5), these measurements clearly exhibited a reduction in conductivity with increasing annealing time that can also be associated with the reduction in the real part of the complex conductivity in DEA measurements (Fig. 9).

\subsubsection{Temperatures above $T_{\mathrm{c}}$}

Above the critical temperature and at the final stage (long annealing times), cable sample conductivity is noticeably higher than conductivity for XLPE cylinders (copper electrodes). Within this range of temperatures, hopping conduction processes between traps are superimposed on those based on electronic band transport. The increment in the concentration of traps has two different sources. On the one hand, we must consider ions diffused from SC screens and, on the other hand, ions provided by crosslinking by-products decomposition.

Concerning copper electrodes samples, the first-and most important-source of ions is not available, which explains quite obviously their corresponding smaller values of conductivity as observed in Figs. 6-8. Furthermore, curves depicted in these figures show that, initially, during the very first hours of annealing, the variation of conductivity versus annealing time is similar for both types of electrodes: it begins with a decrease. We should first take into consideration that significant diffusion of components from semiconductor through insulation volume requires a certain time and, secondly, that the higher the temperature, the more efficient the diffusion process becomes. In this way, the process that initially appears is electronic band conduction and consequently, as we remarked in last section $\left(T_{\mathrm{a}}<T_{\mathrm{c}}\right)$, the conductivity diminishes with annealing time. After a certain annealing time, in the case of cable samples, ion generation-both from crosslinking byproducts and diffused components from SC screensbecomes an effective process and a noticeable increase of conductivity with annealing time results. This trend is indeed due to the increment of ions inside the XLPE insulator, which enhances hopping conduction between them. Finally, this referenced conductivity increase does not continue indefinitely, but rather it reaches a saturation level. Our experimental results are compatible with previous publications in the literature [21] and with the theoretical model of Nath et al. [20], although our results indicate that the parameter associated with the distance between localized states should be a time and temperaturedependent one, because it is conditioned by the density of defects, which changes with the diffusion process during annealing procedures.

In the case of XLPE cylinders (copper electrodes), the generation of ions is restricted to the source from crosslinking by-products, whereas this is the process responsible for conductivity growing after its initial decrease. Once this source of ions is exhausted, and taking into account that many of these generated species are volatile, the contribution to the conductivity by hopping between traps progressively diminishes until a nearly constant value is attained. This final regime is based on electronic conduction over extended states and is marked by the material equilibrium structure at the corresponding temperature. The preceding explanations can justify to a great extent two aspects clearly defined in the curves in Figs. 6-8. On the one hand, the higher the temperature, the faster the minimum value in conductivity is reached after the initial decrease. This is due to the fact that high temperatures enhance the effectiveness of diffusion and decomposition of crosslinking by-products, making the process of hoppingconduction between traps more efficient for shorter times. On the other hand (in the case of copper electrodes), the higher the temperature, the faster the conductivity regime of electronic-band transport is achieved (due to the depletion of ions proceeding from crosslinking by-products).

This explanation is also valid for experimental values obtained by DEA. At low frequencies (under $f=0.1 \mathrm{~Hz}$ for $T_{\mathrm{a}}=95^{\circ} \mathrm{C}$ and under $f=1 \mathrm{~Hz}$ for $T_{\mathrm{a}}=100^{\circ} \mathrm{C}$ Figs. 10 and 11) we can observe that initially, the real part of conductivity tends to increase, then it acquires a maximum value and afterwards it diminishes with annealing time. This behaviour is in accordance with the relaxation associated with ions present inside XLPE. They are 
generated by dissociation of by-products so that, temporarily, the contribution of ions is enhanced while annealing time grows. As these elements are mainly volatile, however, they disappear gradually. As a consequence, their contribution to the real part of conductivity progressively decreases and finally, electronic transport processes prevail again, as one can reasonably interpret from Fig. 11 (see curve corresponding to the larger annealing time, $t_{\mathrm{a}}=3$ days). Under this model, the crossover frequency of isochrone curves is produced by the difference in the evolution of conductivity, at high and low frequencies, with annealing time. Furthermore, this crossover frequency is also dependent on $T_{\mathrm{a}}$. The higher the temperature, the higher the crossover frequency. For this reason, a precise determination of $T_{\mathrm{c}}$ by measurements in the frequency domain is much more difficult than its determination in the time domain. As annealing temperature decreases, this crossover frequency decreases as well, and, regrettably, this frequency eventually is lower that the frequencies that can be reached experimentally in our set-up. In Fig. 12, we have represented as a Mott plot $\left(\ln (\sigma)\right.$ versus $\left.T^{-1 / 4}\right)$ the contribution to conductivity of the hopping mechanism between traps in the case of the "complete" cable samples with $\sigma_{\mathrm{H}}=\sigma-\sigma_{\mathrm{EX}}$, where $\sigma$ is the total conductivity and $\sigma_{\mathrm{EX}}$ is the contribution to conductivity by extended states. The values for conductivities were obtained from the quasistationary regimes (saturation values), corresponding to very long annealing times, for different annealing temperatures. A linear behaviour of $\ln \left(\sigma_{\mathrm{H}}\right)$ versus $T^{-1 / 4}$ can be estimated, which implies that the transport mechanism is basically via thermally assisted hopping conduction [32]. The resulting value for conductivity by extended states is $\sigma_{\mathrm{EX}}=1.65 \times 10^{-17} \mathrm{~S} / \mathrm{m}$. Hill [35] also found a similar relation between conductivity and annealing temperature, by considering a field-assisted hopping conductivity at the limit of low electric fields, which is evidently one of the important experimental conditions observed in our present work.

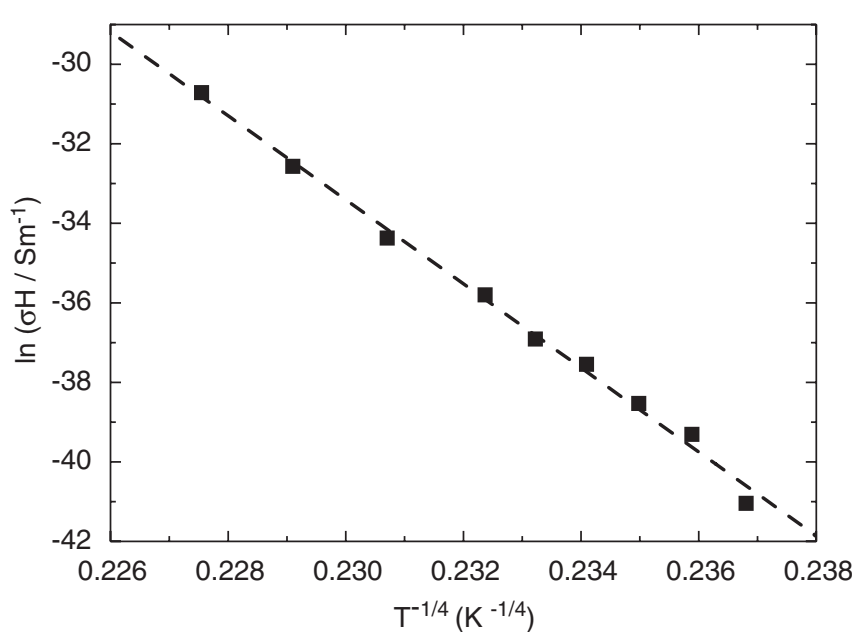

Fig. 12. Mott diagram for representing the contribution to conductivity by hopping processes between localized states.

\section{Conclusions}

We have studied the conductive properties of real MV distribution cables with XLPE insulation and extruded SC screens for a range of annealing temperatures that includes the service temperature range of power distribution cables $\left(\approx 90^{\circ} \mathrm{C}\right)$. We observed significant differences in the behaviour of cable samples with SC screens and without SC screens (XLPE cylinders). SC screens condition very much the electrical behaviour of the entire cable. We conclude that in order to understand the thermoelectrical aging of power cables, it is necessary to take into account the SC screens in both theoretical and experimental research. Furthermore, research devoted both to understand aging/breakdown processes and to improve insulation quality in MV and HV power cables should be carried out on "complete" cable sections, i.e., including extruded SC screens.

Measurements that allowed us to determine the evolution of conductivity with annealing time were performed by using two different methods: absorption/resorption current technique (time domain), and dynamic electrical analysis (frequency domain). We obtained good correlation between results of measurements carried out in both domains (time and frequency), as well as a plausible explanation for them, based on the coexistence of two conduction mechanisms. The first mechanism involves extended states, being slightly dependent on temperature (at least for our temperature interval of interest). The second mechanism operates by carrier hopping between traps and is thermally assisted. This latter mechanism is efficient from a certain critical temperature $T_{\mathrm{c}}$, and it is associated with the creation and diffusion of components, essentially coming from the SC screens. These SC screens generate an increment of ion concentration for temperatures above $T_{\mathrm{c}}$ that involves a significant increase in conductivity with annealing time. The latter can be justified by this second conduction mechanism.

\section{Acknowledgements}

This work has been partially supported by the Spanish Ministry of Science and Technology (Project MAT 2001-2338-C02-01). Authors acknowledge collaboration of company General Cable S.A. through the development of the entire work, in particular by providing power cables and performing several types of treatment on them.

\section{References}

[1] P.H. Lindenmeyer, V.F. Holland, Relationship between molecular weight, radial growth rate and the width of the extinction bands in polyethylene spherulites, J. Appl. Phys. 35 (1964) 55-58.

[2] P.J. Phillips, Morphology - electrical property relations in polymers, IEEE Trans. Electr. Insul. 13 (1978) 69-81.

[3] J. Muccigrosso, P.J. Phillips, The morphology of cross-linked polyethylene insulation, IEEE Trans. Electr. Insul. 13 (1978) $172-178$. 
[4] G.A. Cartwright, A.E. Davies, S.G. Swingler, A.S. Vaughan, IEEE Proc. Sci. Meas. Technol. 143 (1996) 26-34.

[5] A. Campus, B. Lafin, T. Sterzynski, Structure morphology modification of cable insulation polymers, In: Proceedings of the Sixth IEEE ICSD, Vasteras, Sweden, June, 1998.

[6] J.C. Fothergill, G.C. Montanari, G.C. Stevens, C. Laurent, G. Teyssedre, L.A. Dissado, U.H. Nilsson, G. Platbrood, Electrical, microstructural, physical and chemical characterization of HV cable peelings for an electrical ageing diagnostic database, IEEE Trans. Electr. Insul. 10 (2003) 514-527.

[7] X. Wang, N. Yoshimura, D. Tu, Y. Tanaka, T. Takada, Distribution characteristics and formation mechanism of space charge in PE materials, In: Proceedings of the 1998 International Symposium on Electrical Insulating Materials, Toyohashi, Japan, September 1998.

[8] K. Yahagi, Dielectric properties and morphology in polyethylene, IEEE Trans. Electr. Insul. 15 (1980) 241-250.

[9] L.A. Dissado, J.C. Fothergill, Electrical Degradation and Breakdown in Polymers, Peter Peregrinus Ltd, London, 1992.

[10] J.N. Marat-Mendes, R.M. Neagu, E.R. Neagu, Electrical conduction and space charge trapping in highly insulating materials, J. Phys. D: Appl. Phys. 37 (2004) 343-347.

[11] A. Cavallini, D. Fabiani, G. Mazzanti, G.C. Montanari, Life model based on space-charge quantities for HVDC polymeric cables subjected to voltage-polarity inversions, IEEE Trans. Diel. Electr. Ins. 9 (2002) 514-523.

[12] A. Cavallini, D. Fabiani, G. Mazzanti, G.C. Montanari, L. Simoni, Life estimation of DC insulation systems in the presence of voltage-polarity inversions, In: Conference Records of the 2000 IEEE ISEI, Anaheim, USA, April 2000.

[13] P. Notingher, A. Toureille, J. Santana, L. Martinotto, M. Albertini, Study of space charge accumulation in polyoleofins submitted to AC stress, IEEE Trans. Diel. Electr. Ins. 8 (2002) 972-984.

[14] N. Zebouchi, T.G. Hoang, A. Bui, Termoelectronic breakdown with pressure and space charge effects in PE, J. Appl. Phys. 81 (5) (1997) 2363-2369.

[15] K.C. Frisch, A. Patsis, in: D.A. Seanor (Ed.), Electrical Properties of Polymers, Academic Press, New York, 1982.

[16] M.A. Lampert, P. Mark, Current Injection in Solids, Academic Press, New York, 1970.

[17] S. Pélissou, H. St-Onge, M.R. Wertheimer, Electrical conduction in low density polyethylene, IEEE Trans. Electr. Insul. 23 (1988) $325-332$.

[18] G. Chen, H.M. Banford, R.A. Fouracre, D.J. Tedford, Electrical conduction in low density polyethylene, In: Proceedings of the Third Conference on Conduction and Breakdown in Solids, Trondheim, Norway, July 1989.

[19] T. Mizutani, I.B. Jordan, Electrical conduction of PE, In: Annual Report of the 1974 Conference on Electrical Insulation and Dielectric Phenomena, Downington, USA, October 1974.

[20] R. Nath, T. Kaura, M.M. Perlman, Steady-state conduction in linear low-density polyethylene with Poole-lowered trap depth, IEEE Trans. Electr. Insul. 25 (1990) 419-425.
[21] K.R. Bambery, R.J. Fleming, J.T. Holboll, Space charge profiles in low density PE samples containing a permittivity/conductivity gradient, J. Phys. D: Appl. Phys. 34 (2001) 3071-3077.

[22] N. Hozumi, T. Takeda, H. Suzuki, T. Okamoto, Space charge behavior in XLPE under $0.2-1.2 \mathrm{MV} / \mathrm{cm}$ dc fields, IEEE Trans. Diel. Electr. Insul. 5 (1998) 82-90.

[23] M. Ieda, Electrical conduction and carrier traps in polymeric materials, IEEE Trans. Electr. Insul. EI-19 (1984) 162-177.

[24] I. Tamayo, J. Belana, J.C. Cañadas, M. Mudarra, J.A. Diego, J. Sellarès, Thermally stimulated depolarization currents of crosslinked polyethylene relaxations in the fusion range of temperatures, J. Polym. Sci. Part B Polym. Phys. 41 (2003) 1412-1421.

[25] I. Tamayo, J. Belana, J.A. Diego, J.C. Cañadas, M. Mudarra, J. Sellarès, Space charge studies of crosslinked polyethylene midvoltage cable insulation by stimulated depolarization current, infrared/Fourier transform infrared and scanning electron microscopy, J. Polym. Sci. Part B Polym. Phys. 42 (2004) 4164-4174.

[26] M. Acedo, I. Radu, F. Frutos, J.C. Filippini, A. Jiménez, J.A. PérezGómez, A. Jadraque, The characterization of a LDPE affected by different levels of water tree degradation using absorption current measurements, In: Seventh International Conference on Conduction and Breakdown in Solid Dielectrics, Eindhoven, Holland, June 2001.

[27] P. Werelius, P. Thärning, R. Erikson, B. Holmgren, U. Gäfvert, Dielectric spectroscopy for diagnosis of water tree deterioration in XLPE cables, IEEE Trans. Diel. Electr. Insul. 8 (2001) 27-42.

[28] S. Hvidsten, H. Faremo, J.T. Benjaminsen, E. Ildstad, Condition assessment of water treed service aged XLPE cables by dielectric response measurements, CIGRE paper 21-201, CIGRE: Paris, 2000.

[29] F. Frutos, M. Acedo, A. Jadraque, M. Mudarra, J. Sellarès, J. Belana, Comparative study of conductivity in mid-voltage cable XLPE insulation, In: International Conference on Solid Dielectrics, Toulouse, France, July 2004.

[30] M. Acedo, Relación entre la degradación del polietileno por arborescencias de agua y las propiedades eléctricas del material. Aplicación al diagnóstico del aislamiento de los cables de distribución de energía. Ph.D. Thesis, Universidad de Sevilla, Sevilla, Spain, February 2004.

[31] I. Tamayo, Estudio del comportamiento de la carga de espacio durante la fusión del XLPE en cables de media tensión por TSDC. Ph.D. Thesis, Universitat Politècnica de Catalunya, Terrassa, Spain, May 2002.

[32] N.F. Mott, E.A. Davis, Electronic Processes in Non-Crystalline Materials, Oxford University Press, Oxford, 1979.

[33] H. Herman, J. Thomas, G. Stevens, Spectroscopic and chemometrics analysis of cable condition in the Artemis program, In: International Conference on Solid Dielectrics, Toulouse, France, July 2004.

[34] J.A. Diego, J. Belana, J. Òrrit, J. Sellarès, M. Mudarra, J.C. Cañadas, TSDC study of XLPE recrystallization effects in the fusion range of temperatures, J. Phys. D: Appl. Phys. 39 (2006) 1932-1938.

[35] R.M. Hill, Hopping conduction in amorphous solids, Philos. Mag. 23 (1971) 1307-1325. 